\title{
Estilos de humor en universitarias dominicanas
}

\author{
Humor styles in dominican university students
}

\author{
Ureña Ramírez, Ana Jacqueline, ${ }^{1}$ Ph.D, Urbáez Echavarria, Nancy Josefina, ${ }^{1}$ Ph.D, \\ Galiana Llinares, Laura, ${ }^{2}$ Ph.D, Oliver Germes, Amparo, ${ }^{2}$ Ph.D.
}

${ }^{1}$ Universidad Autónoma de Santo Domingo, Facultad de Psicología. República Dominicana. ${ }^{2}$ Universidad de Valencia (España). Correspondencia: aurena04@uasd.edu.do

Recibido: 28-09-2016; Aceptado: 05-12-2016

\section{RESUMEN}

A la luz de las implicaciones de la psicología positiva, recientemente se ha dedicado atención al estudio del humor y su relación con la salud destacando sus beneficios para las personas. Esta conceptualización como "humor terapéutico" ha promovido investigaciones (Cann y Etzel, 2008; Capps, 2006; Erickson y Feldstein, 2007; Kuiper, Martin y Olinger, 1993; Martin et al., 2003; Vilaythong, Aranu, Rosen y Mascaro, 2003) que han derivado en la elaboración de diferentes escalas del humor, determinando que existen diferentes estilos del mismo. Algunos estilos de humor tienen claras raíces en la historia y cultura dominicana como es discutido en este trabajo. El objetivo de esta investigación es estudiar los diferentes estilos de humor, su relación con la edad, con la felicidad subjetiva y con la depresión en una muestra de 145 universitarias dominicanas. Se utilizan instrumentos como el Cuestionario de Estilos de Humor (HSQ) (Martin et al., 2003), la Escala de Depresión del Centro para Estudios Epidemiológicos (Kohout, Berkman, Evans y Cornoni-Huntley, 1993) y la Escala de Felicidad Subjetiva (Lyubomirsky y Lepper, 1999). Los resultados muestran diferencias entre los diversos estilos de humor con excepción del humor agresivo y de descalificación que resultan semejantes en su intensidad de manifestación: el humor de mejora personal es el menos prevalente, seguido del afiliativo y ambos son significativamente menores en promedio que el resto, los nocivos. Las jóvenes dominicanas, arrojan al igual que poblaciones descritas en literaturas como la española, un perfil con predominancia de humor agresivo y de descalificación sobre el uso del humor afiliativo y de mejora personal. Se observa un comportamiento diferencial con la depresión del humor agresivo y el descalificativo: solo el segundo es significativo, y tiene signo negativo. Los cuatro estilos de humor se muestran independientes de la edad.

Palabras clave: estilos de humor, depresión, felicidad subjetiva, cultura dominicana. 


\section{ABSTRACT}

In light of the implications of positive psychology, attention has recently been devoted to the study of humor and its relation to health, highlighting its benefits for people. This conceptualization as a "therapeutic humor" has promoted research (Cann and Etzel, 2008, Capps, 2006, Erickson and Feldstein, 2007, Kuiper, Martin and Olinger, 1993, Martin et al., 2003, Vilaythong, Aranu, Rosen and Mascaro, 2003) that have resulted in the elaboration of different scales of humor, determining that there are different styles of the same. Some humor styles have clear roots in Dominican history and culture as discussed in this paper. The objective of this research is to study the different styles of humor, its relationship with age, with subjective happiness and with depression in a sample of 145 Dominican university students. Instruments such as the Humor Styles Questionnaire (HSQ) (Martin et al., 2003), the Center Depression Scale for Epidemiological Studies (Kohout, Berkman, Evans and Cornoni-Huntley, 1993) and the Subjective Happiness Scale (Lyubomirsky and Lepper, 1999). The results show differences between the different styles of humor with the exception of aggressive humor and disqualification that are similar in their intensity of manifestation: the humor of personal improvement is the less prevalent, followed by the affiliative and both are significantly lower on average than the rest, the harmful ones. The young Dominicans, as well as populations described in literatures such as the Spanish, throw a profile with predominance of aggressive humor and disqualification on the use of affiliative humor and personal improvement. A differential behavior is observed with the depression of the aggressive humor and the disqualifying one: only the second one is significant, and it has negative sign. The four mood styles are shown independent of age.

Keywords: humor styles, depression, subjective happiness, Dominican culture.

\section{INTRODUCCIÓN}

Una novedosa e interesante línea de investigación se ha estado desarrollando en los últimos tiempos, para establecer la relación entre humor y salud. Una de las principales preocupaciones de la literatura que relacionan el humor y la salud (p.e. Lillo, 2006; Cayssials, D'Anna, Pérez, 2006; Carbelo, 2008) consiste en cómo definir el concepto de humor desde el punto de vista terapéutico.

Una de las principales contribuciones a este tema establece beneficios del humor terapéutico como que: "facilita la comunicación y la empatía entre el paciente y el profesional de salud y crea el clima apropiado para la expresión de emociones, preguntas y miedos" (Carbelo, 2008, p.31). La autora puntualiza que el humor efectivo a nivel de salud es "el humor capaz de proveer un ambiente lleno de risas sanas que ayudan a manejar el stress, lograr la adaptación sin ofensas ni subvaluaciones" (Carbelo, 2008, p. 30). Cayssials et al (2006) señalan que la creencia popular sostiene que el humor es saludable y promueve el bienestar de las personas. En este último trabajo, se incluyen aspectos especialmente relevantes para la presente investigación, como la distinción entre tipos de humor apropiados e inapropiados. En concreto, se menciona que son potencialmente beneficiosos para el bienestar los estilos afiliativo y de mejora personal. Efectivamente, estos dos estilos de humor podrían considerarse apropiados, y los otros dos se presentan como estilos que pueden ir en detrimento del bienestar, tanto de quienes lo utilizan como de quienes lo rodean, esto es, podrían considerarse inapropiados éticamente. De manera más específica este estudio considera 
que no todo el humor es terapéutico, y que tan solo el humor benigno o positivo, que incluye los dos factores citados, puede considerarse terapéutico.

Se ha indicado que este humor terapéutico produce los siguientes beneficios a nivel de la salud: estimula el sistema inmunológico y combate el stress (Berk et al., 1989); estimula la secreción de endorfinas, las cuales tienen un efecto analgésico (Yoshino et al., 1996; Weeks, 2000); incrementa la sensación o sentimiento de bienestar (Black, 1984; Fry, 1992); incrementa la autoestima (Porterfield et al. 1988); y distrae los síntomas del dolor (Matz and Brown, 1998). De esta misma forma se ha encontrado que el sentido del humor tiene efectos beneficiosos en la salud y en el bienestar (Kuiper y McHale, 2009; Lefcourt, 2001; Martin, 2001; Sirigatti, Penzo, Giannetti, Casale, y Stefanile, 2016; Tucker et al., 2013; Yue, Liu, Jiang, y Hiranandani, 2014; Zhao, Kong, y Wang, 2012; Zhao, Wang, y Kong, 2014).

Los investigadores, con el interés de identificar las relaciones entre todos los factores o estilos de humor y las variables de salud relacionadas, han desarrollado varios instrumentos de autoevaluación a través de los años. Ejemplos de estos autoinformes son Svebak's Sense of Humor Questionnaire (Svebak, 1974a, 1974b), Situational Humor Response Questionnaire (SHRQ; Martin y Lefcourt, 1984), StateTrait Cheerfulness Inventory (Ruch, Willibald, y Jennifer Hofmann, 2012; Ruch, Willibald, Gabriele Köhler y Christoph van Thriel, 1996). Sin lugar a dudas, uno de los instrumentos más utilizados para evaluar por qué las personas utilizan el humor diariamente de manera espontánea en sus vidas es el Humor Styles Questionnaire (HSQ; Martin, Phulik-Doris, Larsen, Gray, y Weir, 2003). El HSQ responde a los intereses de esta investigación porque es capaz de distinguir entre el humor positivo y negativo. Aparentemente, hay un tipo de humor que influye en la salud de manera positiva y hay otro tipo de humor que puede serle dañino.

EI HSQ es una herramienta de medida diseñada para evaluar los estilos de comportamientos habituales relacionados con el humor. Martin et al. (2003) identificaron cuatro patrones del humor en un continuo de dos direcciones, uno "el humor para fomentar el desarrollo del yo versus las relaciones con los otros" y en la otra "humor benigno versus humor potencialmente detrimente" Martin et al. (2003).

Desde este continuo fueron identificados dos estilos de humor positivos y dos negativos: (a) Humor de afiliación, características del individuos que dice cosas graciosas o chistes para facilitar las relaciones interpersonales de manera benigna; (b) autodesarrollo del humor o humor de mejora personal, involucra una mirada humorística de la vida para alegrarse uno mismo (Kuiper et al., 1993); c) humor agresivo, relacionado con el uso del sarcasmo, la ridiculez y de molestar a los otros para destacarse a costa de ellos; y (d) humor de auto-defensa descalificador personal o autodescalificador, centrado en la intención de divertir a otros mientras se auto ridiculiza (Martin et al., 2003). Evidencia sobre esta estructura del humor de cuatro dimensiones ha sido recabada en diferentes culturas y poblaciones: canadiense (Martin et al., 2003), argentina (Lillo, 2006), libanesa (Taher, Kazarian \& Martin, 2008); italiana (Sirigatti, Penzo, Giannetti \& Stefanile, 2014), austriaca y alemana (Stieger, Formann, \& Burger, 2011), israelita (Zeigler-Hill \& Besser, 2011), china (Yue et al., 2014; Zhao et al., 2012, 2014), o taiwanesa (Chiang, Sheng, Wu, \& Yen, 2014).

A lo largo de estos estudios, los estilos de humor se han relacionado con el sexo y la edad de forma sistemática. Por ejemplo, en el estudio de Martin et al. (2003), participantes jóvenes alcanzaron mayores puntuaciones en 
el humor afiliativo y agresivo que los participantes de mayor edad. Este resultado parece indicar que las personas con más edad, se involucran en hacer bromas o reír con otros practicando un humor afiliativo. Así mismo utilizan menos el humor para menospreciar, manipular o ridiculizar a otros. En esta etapa, el humor se utiliza menos para el beneficio propio o para las relaciones con otros. Tal como Martin et al. (2003) establecen, ello puede deberse a una menor implicación en las actividades sociales.

Al analizar las relaciones entre humor y sexo Martin et al. (2003) y Sirigatti et al. (2014) encontraron puntuaciones más altas del estilo de humor mal adaptado en los hombres, mientras que las mujeres encontraron puntuaciones más altas en el estilo de humor más adaptativo. Estos resultados se corresponden con las tendencias de otras investigaciones donde indican que los hombres se involucran más en estilos de humor de corte inadaptado en comparación con las mujeres (Crawford y Gressley, 1991; Martin y Kuiper, 1999).

A pesar del amplio uso del HSQ, no se conocen hasta el momento datos en ningún país del Caribe, siendo un único estudio el llevado a cabo en América Latina (Lillo, 2006). El humor propiamente dicho no ha sido muy estudiado en la República Dominicana, aunque se han identificado algunos autores (Pérez, 1981 y 2016; Zaglul, 2015) que han expresado sus reflexiones sobre el humor dominicano a través de su interacción con esta población. El objetivo de la presente investigación es estudiar de manera exploratoria la presencia de los estilos de humor según el HSQ en una muestra de universitarias dominicanas, así como aportar evidencia de su relación con la edad y dos importantes criterios de salud o bienestar como son la depresión y la felicidad subjetiva en este particular contexto de la región Caribe. Este primer estudio servirá de base para futuras investigaciones en las cuales se incluirán otras variables que permitirán elevar conclusiones a nivel transcultural.

\section{METODOLOGÍA}

Diseño, procedimiento y muestra. Dadas las características exploratorias de este estudio, se realizó un muestreo intencional en el que participaron inicialmente, en este estudio transversal, un total de 364 estudiantes universitarios de la Universidad Autónoma de Santo Domingo UASD (República Dominicana), de los cuales un $91.3 \%$ fueron mujeres. Los criterios de inclusión fueron ser de sexo femenino y haber completado la totalidad de secciones y variables. Así, se consideraron válidos únicamente aquellos respondidos completamente, y dado el perfil mayoritariamente femenino del colectivo estudiantil encuestado, la muestra final del estudio la conformaron 145 estudiantes universitarias. Su media de edad fue de 28.06 años (DT $=6.59$ ). En cuanto a los estudios, la mayoría (83.4\%) cursaba estudios de Psicología, y las restantes participantes de otras áreas, como Bioanálisis, Economía, Ingeniería, Humanidades o Derecho.

Completaron el cuestionario de forma anónima mediante la plataforma online Survey Monkey. El estudio fue aprobado por el Comité de Ética para la Investigación en Humanos de la Universitat de València (España) (referencia H1460029625650).

Instrumentos. Para llevar a cabo esta investigación se utilizaron medidas de estilos de humor, de felicidad subjetiva y de depresión. El Cuestionario de Estilos de Humor (Humor Styles Questionnaire, HSQ; Martin et al., 2003). El HSQ está formado por 32 ítems, distribuidos igualmente en cuatro subescalas cuya puntuación global se calcula por suma: humor afiliativo, humor de mejora personal, humor agresivo y humor de descalificación personal. Los ítems puntúan en una escala tipo Likert de 7 anclajes, que oscilan de 1 (totalmente en desacuerdo) a 7 (totalmente de 
acuerdo). En esta aplicación el a de la escala global fue de .77 , el de la subescala de humor afiliativo de .68, de mejora .56, agresivo .48 y descalificativo .76 .

También se recabaron datos sobre depresión con la escala de 10 items del Centro para Estudios Epidemiológicos (CES-D 10; Kohout, Berkman, Evans, y Cornoni-Huntley, 1993). Se trata de un cuestionario de autoinforme que aborda síntomas depresivos experimentados en la semana anterior a la administración de los cuestionarios. La puntuación global valora la gravedad y frecuencia de síntomas depresivos. Los valores van desde 0 (raramente o menos de un día) a 3 (siempre/5 a 7 días por semana). La consistencia interna por alfa fue de .77. La Escala de Felicidad Subjetiva (SHS; Lyubomirsky y Lepper, 1999). Son 4 items que valoran felicidad general con anclajes tipo Likert de 7 puntos desde 1 (no, en absoluto) a 7 (completamente). La consistencia interna fue de .78.

Análisis de datos. Para estudiar los estilos de humor en el contexto de estudiantes universitarias, mayoritariamente de psicología, de la Universidad Autónoma de Santo Domingo de la República Dominicana, se calcularon medias y desviaciones típicas para la muestra, y se realizó después un ANOVA intra donde se tomó la edad como posible covariable, y por tanto se controló su efecto. Se examinó la relación de los estilos de humor con la edad y variables clave como felicidad y depresión. En el primer caso, se llevó a cabo un análisis de varianza de medidas repetidas con edad como covariable (ANOVA). En el segundo, se obtuvieron correlaciones de Pearson entre la edad y los estilos de humor.

\section{RESULTADOS}

Manifestación de los estilos de humor. Las medias y desviaciones típicas de cada una de las dimensiones de los estilos de humor para facilitar la comprensión delosresultados, seaportan en la tabla 1. El ANOVA intra con edad como covariable cumplió los supuestos requeridos en cuanto a esfericidad (epsilon de Huyhn y Feldt $=.843$ ) y reveló efecto no significativo ni con tamaño de efecto apreciable de la covariable edad. En cuanto al análisis principal, para esclarecer si existen diferencias significativas entre las 4 dimensiones, el análisis fue claro en el sentido de que sí se detectan diferencias y de tamaño del efecto grande $(F(3,411)=8.62$; $\left.p<.001, h^{2}=.059\right)$. Consecuentemente, las pruebas complementarias de ajuste de Bonferroni realizadas ofrecieron evidencia sobre las diferencias entre casi todas las dimensiones. Únicamente no resultaron diferentes entre sí las dimensiones humor agresivo y humor basado en la descalificación. Todas las demás lo fueron con un valor $\mathrm{p}<.001$, salvo la diferencia entre el humor afiliativo y el segundo, humor de mejora personal, con $p=.014$.

Esta evidencia sobre estilos de humor puede ser comparada frente a los valores obtenidos en su aplicación española (tabla 1), se observa su similitud.

Tabla 1. Medias y desviaciones típicas en las cuatro subescalas del cuestionario de estilos de humor en comparación con resultados aplicación española.

\begin{tabular}{ccc}
\hline & R. Dominicana & España \\
\hline Afiliativo & $25.57(7.75)$ & $23.47(9.78)$ \\
Mejora personal & $23.65(6.51)$ & $26.82(6.81)$ \\
Agresivo & $40.08(7.35)$ & $38.64(8.05)$ \\
Descalificativo & $40.48(8.55)$ & $37.76(9.47)$ \\
\hline
\end{tabular}

La tabla 1 presenta la comparación de los resultados de la media y DT entre la muestra dominicana y la española, y las diferencias entre ellas son mínimas. En sentido general estas variaciones oscilan entre $2 \%$ y $3 \%$ en cada uno de los diferentes tipos de humor.

Relación con la edad, la depresión y la felicidad. Además de los análisis recién presentados, se exploraron las relaciones entre estas 4 dimensiones o estilos de humor, la edad (ningún valor 
resultó significativo), la depresión y la felicidad subjetiva.

Tabla 2. Correlaciones entre estilos de humor, edad, felicidad y depresión

\begin{tabular}{ccccc}
\hline & Afiliativo & $\begin{array}{c}\text { Mejora } \\
\text { personal }\end{array}$ & Agresivo & Descalificativo \\
\hline Felicidad & -.146 & $-.222^{* *}$ & .012 & .090 \\
Depresión & .097 & $.195^{*}$ & -.115 & $-.259 * *$ \\
Edad & -.070 & -.099 & .032 & .089 \\
$\begin{array}{c}\text { Afiliativo } \\
\begin{array}{c}\text { Mejora } \\
\text { personal }\end{array}\end{array}$ & $.434 * *$ & $.193^{*}$ & .088 \\
Agresivo & & & $.228^{* *}$ & $.281^{* *}$ \\
\hline Nota: $* p<.05 ; * * p<.01$. & & & $.527 * *$ \\
\hline
\end{tabular}

En la tabla 2 se pueden observar los valores de correlación. La medida subjetiva de felicidad no se muestra un criterio sensible ante los diferentes estilos de humor, tan solo se detecta una relación significativa, pero baja en intensidad con el humor de mejora personal (-.222). Los valores de correlación más altos corresponden a la vinculación entre las dimensiones humor agresivo y por descalificación (.527). Los resultados muestran la diferente relación que con la depresión mantienen el humor descalificativo y el agresivo, siendo significativo y de signo negativo en el primer caso (-.259), pero muy baja y no alcanzando la significatividad en el segundo.

\section{DISCUSIÓN Y CONCLUSIONES}

El objetivo fundamental de esta investigación consistió en estudiar la presencia de los estilos de humor (afiliación, humor de mejora personal, humor agresivo y humor de autodefensa) y su posible relación con la edad, la felicidad subjetiva y la depresión, en una muestra de estudiantes universitarias en República Dominicana. Un objetivo secundario fue, a la luz de estos resultados, compararlos con otro contexto de habla hispana, como es población española universitaria estudiada recientemente bajo estos mismos cuatro tipos de humor. En este sentido, jóvenes-adultas dominicanas, arrojan como las españolas, un perfil con predominancia de humor agresivo y de descalificación, contrario a lo que en la literatura (Cayssials et al., 2006) se identifica como benigno.

Los resultados revelaron la nula influencia de la edad, a diferencia del estudio de Martin et al (2003) que identificaba la relación entre edad y dos de los estilos (afiliativo y agresivo más presente cuanto más jóvenes). Precisamente estos dos estilos de humor (afiliativo y agresivo) en el presente estudio se han revelado también independientes de los criterios de felicidad y depresión considerados. Sin embargo, el estilo de descalificación ha mostrado una relación significativa de carácter moderado y negativo con depresión; y el de mejora personal con ambos criterios (relación baja pero positiva con depresión y negativa pero algo más intensa con felicidad). Estos resultados pueden ser de interés para guiar el diseño de intervenciones, puesto que indican que estilos de humor conectarían más con la idea de humor terapéutico, orientado al bienestar.

Además, las medias comparadas entre las poblaciones españolas y dominicanas presentan similitudes en todos los tipos de humor, lo que indica la ausencia de efectos explicables por el contexto cultural.

Estos hallazgos pueden confrontarse con estudios de humor realizados en la República Dominicana donde, de alguna manera, la tristeza, la visión pesimista de la vida por parte del dominicano pueda influir. Un ejemplo de esto sería cómo los habitantes aborígenes para transmitir sus historias usaban canciones o romances, asumidos de la cultura española, donde se burlaban de los españoles con "cantaletas" (Guerrero, 2005). Más recientemente, el psiquiatra dominicano Antonio Zaglul (2015) reseña: "Nuestro humor es seco y espasmódico; llevamos dentro de nosotros lo trágico del español y la tristeza del negro esclavo. ... Tenemos 
un sentido exagerado del ridículo y reír es hacer el ridículo para nosotros" (pág. 84). En este mismo sentido Pérez (1981) refiere: "nadie puede negar que este es un país donde continuamente se pone de manifiesto un humor seco y cortante". Esta información ubica la cultura dominicana próxima a categorías de humor agresivo y auto descalificador. Este planteamiento es de total actualidad, pues como corrobora la prensa dominicana en el artículo titulado Benéfico humor dominicano (2016) los humoristas dominicanos se inspiran en gran medida en la "tendencia del criollo a burlarse de sus desventuras y de las ajenas". Este tipo de humor, como apuntan los resultados del presente trabajo, se relaciona con la depresión, entre otros aspectos (Cassaretto y Martínez, 2009). En consonancia con esta afirmación, para Zaglul (2015) "no solo es triste el dominicano; también es malhumorado, es una tristeza irritable casi permanente" (pág. 85). Por otro lado, se considera al dominicano muy sensible a las críticas y a las bromas, posiblemente por su idealismo y aislamiento, cuando se trata sobre su país, sus costumbres o revoluciones (Schoennrich, 1977, en Sánchez, 1997). En República Dominicana, se utilizaría el humor como una forma de afrontamiento ante los eventos estresantes propios de un país en desarrollo, en la línea de los trabajos de Frankl (1991).

En definitiva, este trabajo ofrece una primera evidencia en el contexto de la región del Caribe, mostrando en universitarias dominicanas diferencias entre los diversos estilos de humor, con excepción del humor agresivo y de descalificación, que resultaron ser semejantes en su intensidad de manifestación.

Conscientes de las limitaciones de este trabajo, que implícitamente apuntan a líneas futuras como estudiar su replicabilidad en muestras mayores y de ambos sexos, se ha intentado aportar una primera evidencia científica en jóvenes-adultas dominicanas sobre un importante precursor de calidad de vida. Este primer diagnóstico, simplemente abre la vía para su estudio y más a largo plazo el diseño y evaluación de intervenciones que, usando los estilos de humor apropiados como herramienta, promuevan la salud y el bienestar en la región del Caribe. Este estudio pretende aportar desde la psicología positiva, una alternativa más, para proveer bienestar a la población y contribuir a su salud mental, en un contexto especialmente vulnerable, tal como indican informes recientes (Comisión Económica para América Latina y Caribe, 2016).

\section{REFERENCIAS}

Bell, N. D. (2007). Humor comprehension: Lessons learned from cross-cultural communication. Humor. International Journal of Humor Research, 20, 4, 367387.

Bentler, P. M., \& Bonett, D. G. (1980). Significance tests and goodness of fit in the analysis of covariance structures. Psychological Bulletin, 88, 588-606.
Bergman, L.R., \& Wångby, M. (2014). The person-oriented approach: A short theoretical and practical guide. Estonian Journal of Education, 2, 29-49. http:// dx.doi.org/10.12697/eha.2014.2.1.02b.

Berk, L. (1989). Neuroendocrine and stress hormone changes during mirthful laughter. American Journal Medicines Science, 298, 390-396. 
Black, D.W. (1984). Laughter. Journal of American Medical Association. JAMA, 252, 2995-2998

Bollen \& H. S. Long (Eds.), Testing structural equation models ( $\mathrm{pp}$. 136162). Beverly Hills, CA: Sage.

Browne, M. W., \& Cudeck, R. (1993). Alternative ways of assessing model fit. In C. A.

Cann, A. \& Etzel, K. (2008). Remembering and anticipating stressors: Positive personality mediates the relationship with sense of humor: International Journal of Humor Research, 2(2),157-178.

Capps, D. (2006). The psychological benefits of humor. Pastoral Psychology, 54(5), 393-411.

Carbelo, B. (2008). El humor en la relación con el paciente. Una guía para profesionales de la salud. Barcelona: Elsevier-Masson.

Cassaretto, M. \& Martínez, P. (2009). Validación de la escala del sentido del humor en estudiantes universitarios. Revista de Psicología, 27, 2, 287-310.

Cayssials, A., D'Anna, A. \& Pérez, M. A. (2006). Estilos del sentido del humor. Un estudio transcultural en población adulta según género. XIII Jornadas de Investigación y Segundo Encuentro de Investigadores en Psicología del Mercosur. Facultad de Psicología - Universidad de Buenos Aires, Buenos Aires. Disponible en http://www.aacademica.org/000$\underline{039 / 547}$

Cheek, J. M. (1983). The Revised Cheek and Buss Shyness Scale (RCBS). Unpublished manuscript, Wellesley College, Wellesley, MA.

Chiang, Y., Sheng, H., Wu, W., \& Yen, L. (2014). Clusters of humor expression and related factors among junior high school students in Taiwan. Bulletin of Educational Psychology, 46(2), 289-310.
Comisión Económica para América Latina y el Caribe, CEPAL (2016). Panorama social de América Latina 2015, (LC/G.2691-P), Santiago, 2016.

Crawford, M., \& Gressley, D. (1991). Creativity, caring, and context: Women's and men's accounts of humor preferences and practices. Psychology of Women Quarterly, 15, 217-231.

Critchley, S. (2002). On humour. London: Routledge.

Darwin, C. (1872) The expression of the emotions in man and animals. London: Murray.

Diener, E., Emmons, R. A., Larsen, R. J., \& Griffin, S. (1985). The satisfaction with life scale. Journal of Personality Assessment, $49,71-75$.

Dyck, K.T.H., \& Holtzman, S. (2013). Understanding humor styles and well-being: The importance of social relationships and gender. Personality and Individual Differences, 55, 53-58. http:// dx.doi.org/10.1016/j.paid.2013.01.023.

Erickson, S. \& Feldstein, S. (2007). Adolescent humour and its relationship to coping, defense strategies, psychological distress, and well-being. Child Psychiatry and Human Development, 37, 255-271.

Frankl, V.E. (1991) El Hombre en Busca de Sentido. Barcelona: Editorial Herder.

Freud, S. (1963). Jokes and their relation to the unconscious. Standard edition of the complete psychological works of Sigmund Freud, vol. 5, trans. J. Strachey. New York: W.W. Norton.

Freud, S. (1987). Humor. trans. J. Rivière. In J. Morreall (Ed.), The philosophy of laughter and humor (pp. 111-116). Albany: State University of New York Press. 
Fry, W.F. (1992). The physiologic effects of humor, mirth, and laughter. Journal of American Medical Association. JAMA, 267, 1857-1858.

Graham, J. M. (2006). Congeneric and essentially tau-equivalent estimates of score reliability: What they are and how to use them. Educational and Psychological Measurement, 66, 930-944.

Hobbes, T. (1839). Leviathan. In The English works of Thomas Hobbes, ed. W. Molesworth, vol. 3. London: Bohn.

Hofstede, G., \& Hofstede, G. J. (2005). Cultures and Organizations (2nd ed.). New York: McGraw Hill.

Kant, I. (1892). Critique of Judgment, trans. J.H. Bernard. London: Macmillan.

Kohout, F. J., Berkman, L. F., Evans, D. A., \& Cornoni-Huntley, J. (1993). Two shorter forms of the CES-D depression symptoms index. Journal of Aging and Health, 5, 179-193.

Kuiper, N. A., \& McHale, N. (2009). Humor styles as mediators between selfevaluative standards and psychological well-Being. The Journal of Psychology, 2009, 143(4), 359-376.

Kuiper, N. A., Martin, R. A., \& Olinger, L. J. (1993). Coping humour, stress, and cognitive appraisals. Canadian Journal of Behavioural Science, 25, 81-96.

Kuiper, N.A. (2012). Humor and resiliency: Towards a process model of coping and growth. Europe's Journal of Psychology, 8, 475-491. http://dx.doi.org/10.5964/ ejop.v8i3.464.

Lefcourt, H. M. (2001). Humor: The psychology of living buoyantly. New York: Kluwer Academic Publishers.
Leist, A. K., \& Müller, D. (2013). Humor types show different patterns of selfregulation, self-esteem, and well-being. Journal of Happiness Studies, 14, 551569. http://dx.doi.org/10.1007/s10902012-9342-6.

Lilo, P. R. (2006). Influencia de los estilos de humor sobre las estrategias de afrontamiento en Entre Ríos. Revista Psicodebate: Psicología, cultura y sociedad, 7, 95-118.

Lyubomirsky, S., \& Lepper, H. (1999). A measure of subjective happiness: preliminary reliability and construct validation. Social Indicators Research, 46, 137-155.

Martin, R. A. (2001). Humor, laughter, and physical health: Methodological issues and research findings. Psychological Bulletin, 127, 504-519.

Martin, R. A. (2007). The Psychology of Humor: An integrative approach. Burlington, CA: Elsevier Academic Press.

Martin, R. A., \& Lefcourt, H. M. (1983). Sense of humor as a moderator of the relation between stressors and moods. Journal of Personality and Social Psychology, 45, 1313-1324.

Martin, R. A., \& Lefcourt, H. M. (1984) Situational Humor Response Questionnaire: quantitative measure of sense of humor. Journal of Personality and Social Psychology, 47, 145-155.

Martin, R. A., Phulik-Doris, P., Larsen, G., Gray, J., \& Weir, K. (2003). Individual differences in uses of humor and their relation to psychological well-being: Development of the Humor Styles Questionnaire. Journal of Research in Personality, 37, 48-75.

Martin, R.A., \& Kuiper, N.A.(1999). Daily occurrence of laughter: Relationships with age, gender, and type A personality. Humor: International Journal of Humor Research, 12, 355-384. 
Matz, A. \& S.T. Brown (1998). Humor and pain management. A review of current literature. Journal of Holistic Nursing, 16, 68-75.

Mendiburo-Seguel, A., Pàez, D., \& Martínez-Sánchez, F. (2015). Humor styles and personality: A meta-analysis of the relation between humor styles and the Big Five personality traits. Scandinavian Journal of Psychology, 56, 335-340. http://dx.doi.org/10.1111/sjop.12209.

Morreall, J. (1987). (Ed.), The Philosophy of Laughter and Humor. Albany: State University of New York Press.

Pérez, M. (1981). Estampas dominicanas: Primera entrega. Publicaciones América. Santo Domingo, R.D.

Pérez, M. "Benéfico humor dominicano" Publicado el 17 de Septiembre de 2016 en Periódico El Nacional, Sto. Domingo. http: //elnacional.com.do/beneficohumor-dominicano/

Plato (1978) Philebus 48-50. In The collected dialogues of Plato (ed. E. Hamilton and H. Cairs). Princeton: Princeton University Press.

Porterfield, A.L., F.S. Mayer, K.G. Dougherty, K.E. Kredich, M.M. Kronberg, K.M. Marsee \& Y. Okazaki (1988). Private self-consciousness, canned laughter and responses to humorous stimuli. Journal of Research in Personality, 22, 409-423.

Raykov, T. (2004). Behavioral scale reliability and measurement invariance evaluation using latent variable modeling. Behavioral Therapy, 35, 299-331.

Rosenberg, M. (1965). Society and the adolescent self-image. Princeton, NJ: Princeton University Press.

Ruch, W. (ed.). (1998). The sense of humor: Explorations of a personality characteristic. New York: Mouton de Gruyter.
Ruch, Willibald \& Jennifer Hofmann. (2012). A temperament approach to humor. In Paola Gremigni (ed.), Humor and health promotion (pp. 79-112). New York: Nova Science.

Ruch, Willibald, Gabriele Köhler \& Christoph van Thriel. (1996). Assessing the temperamental basis of the sense of humor: Construction of the facet and standard trait forms of the State-TraitCheerfulness-Inventory - STCI. Humor: International Journal of Humor Research, 9, 303-339.

Sánchez, M. F. (1997). Psicología del pueblo dominicano. Hacia una interpretación de la psicología dominicana ( $p-153,154)$. Santo Domingo, R.D: Editora Universitaria (UASD)

Sirigatti, S., Penzo, I., Giannetti, E., \& Stefanile, C. (2014). The Humor Styles Questionnaire in Italy: psychometric properties and relationships with psychological well-being. Europe's Journal of Psychology, 10(3), 429-450. http:// dx.doi.org/10.5964/ejop.v10i3.682.

Sirigatti, S., Penzo, I., Giannetti, E.,Casale, S., \& Stefanile, C. (2016). Relationships between humorism profiles and psychological well-being. Personality and Individual Differences, 90, 219-224.

Stieger, S., Formann, A. K., \& Burger, C. (2011). Humor styles and their relationship to explicit and implicit selfesteem. Personality and Individual Differences, 50, 747-750. http://dx.doi. org/10.1016/j.paid.2010.11.025.

Svebak, S. (1974a). Revised questionnaire on the sense of humor. Scandinavian Journal of Psychology, 15, 328-331.

Svebak, S. (1974b) A theory of sense of humor. Scandinavian Journal of Psychology, 15, 99-107. 
Taher, D., Kazarian, S. S., \& Martin, R. D. (2008). Validation of the Arabic Humor Styles Questionnaire in a community sample of Lebanese in Lebanon. Journal of Cross-Cultural Psychology, 39(5), 552-564. http://dx.doi. org/10.1177/0022022108321177.

Tucker, R. P., Judah, M. R., O'Keefe, V. M., Mills, A. C., Lechner, W. V., Davidson, C. L., Grant, D. M., \& Wingate, L. R. (2013). Humor styles impact the relationship between symptoms of social anxiety and depression. Personality and Individual Differences, 55, 823-827.

Van den Broeck, A., Vander Elst, T., Dikkers, J., De Lange A., \& De Witte, $\mathrm{H}$. (2012). This is funny: On the beneficial role of self-enhancing and affiliative humour in job design. Psicothema, 24, 1, 87-93.

Vilaythong, A., Arnau, R., Rosen, D. \& Mascaro, N. (2003). Humor and hope: Can humor and hope: Can humor increase hope? Humor: International Journal of Humor Research, 16(1), 79-89.

Weeks, A. (2000). Let's hear it for endorphins! Online newsletter, www. healingpassages.com.

Wittgenstein, L. (1980). Culture and Value (ed. De G.H. Von Wright). Oxford: Blackwell.

Yoshino, S., J. Fujimori \& M. Khoda (1996). Effects of mirthful laughter on neuroendocrine and immune systems in patients with rheumatoid arthritis. Rheumatology, 23, 793-794.
Yue, X. D., Liu, K. W., Jiang, J., \& Hiranandani, N. A. (2014). Humor styles, self-esteem, and subjective happiness. Psychological Reports: Mental \& Physical Health, 115, 517-525. DOI 10.2466/07.02.PR0.115c18z6

Zaglul, A. (2015). Apuntes. Editora Letra Gráfica: Santo Domingo, República Dominicana.

Zeigler-Hill, V., \& Besser, A. (2011). Humor style mediates the association between pathological narcissism and self-esteem. Personality and Individual Differences, 50, 1196-1201. http://dx.doi.org/10.1016/j. paid.2011.02.006.

Zhao, J., Kong, F., \& Wang, Y. (2012). Selfesteem and humor style as mediators of the effects of shyness on loneliness among Chinese college students. Personality and Individual Differences, 52, 686-690.

Zhao, J., Wang, Y., \& Kong, F. (2014). Exploring the mediation effect of social support and self-esteem on the relationship between humor style and life satisfaction in Chinese college students. Personality and Individual Differences, 64, 126-130.

Zimet, G. D., Dahlem, N. W., Zimet, S. G., \& Farley, G. K. (1988). The multidimensional scale of perceived social support. Journal of Personality Assessment, 52, 30-41. 